2. TOYODA, Y.; SHINOHARA, N.; HARABAYASHI, T. y cols.: "Survival and prognostic classification of patients with metastatic renal cell carcinoma of bone". Eur. Urol., 52: 163, 2007.

3. ZISMAN, A.; PANTUCK, A.J.; DOREY, F. y cols.: "Improved prognostication of renal cell carcinoma using integrated staging system". J. Clin. Oncol., 19: 1649, 2001.

4. WHELAN, P.: "The medical treatment of metastatic renal cell carcinoma". EUA Update Series, 1: 237, 2003.

5. SNITOH, H.: "Distant metastases of renal adenocarcinoma". Cancer, 48: 1487, 1981.

6. SUFRIN, G.: "The challenges of renal adenocarcinoma”. Surg. Clin. North Am., 62: 1101, 1982.

7. GARCÍA RODRÍGUEZ, J.; FERNÁNDEZ GÓMEZ, J.M.; JALÓN MONZÓN, A. y cols.: "Metástasis vesical de carcinoma renal". Arch. Esp. Urol., 57: 1123, 2004.

8. RODRÍGUEZ FABA, O.; MARTÍNEZ GÓMEZ, F.J.; PARRA MUNTANER, L. y cols.: "Carcinoma de células renales con metástasis pulmonar y ocular tardías". Arch. Esp. Urol., 57: 545, 2004.

9. PASCUAL MATEO, C.; NIETO GALLO, M.A.; LUJÁN GALÁN, M. y cols.: "Retención urinaria aguda y hematuria secundarias a metástasis peneana por carcinoma renal de células claras". Actas Urol. Esp., 29: 593, 2005.

10. PÉREZ FENTES, D.A.; BLANCO PARRA, M.; TORCEDO CAAMAÑO, V. y cols.: "Carcinoma renal metastático de localización atípica. Revisión de la literatura". Actas Urol. Esp., 29: 621, 2005.

11. KATO, Y.; NUMATA, A.; WADA, N. y cols.: "A case of metastatic renal cell carcinoma to the ovary". Hinyokika Kiyo, 52: 923, 2006.

**12. VALAPPIL, S.V.; TOON, P.G.; ANANDARAM, P.S.: "Ovarian metastasis from primary renal cell carcinoma: Report of a case and review of literature". Gynecol. Oncol., 94: 846, 2004.

13. YOUNG, R.H.; HART, W.R.: "Renal cell carcinoma metastatic to the ovary: A report of three cases enphasizing possible confusion with ovarian clear cell adenocarcinoma". Int. J. Gynecol. Pathol., 11: 96, 1992.

14. FIELDS, S.; LIBSON, E.; LAVIE, O. y cols.: "Renal cell carcinoma metastatic to the ovary. Ultrasound and CT appearance". Clin. Imaging, 20: 42, 1996.

15. VARA, A.; MADRIGAL, B.; VEIGA, M. y cols.: "Bilateral ovarian metastasic from renal cell carcinoma". Acta oncol., 37: 379, 1998.

**16. HAMMOCK, L.; GHORAB, Z.; GÓMEZ-FERNÁNDEZ, C.R.: "Metastatic renal cell carcinoma to the avary: A case report and discussion of differential diagnoses". Arch. Pathol. Lab. Med., 127: 123, 2003.
Casos Clínicos

Arch. Esp. Urol., 61, 4 (537-540), 2008

\section{HEMATOCELE CRÓNICO IMITANDO UN TUMOR TESTICULAR. PRESENTACIÓN DE DOS CASOS}

\section{Concepción Lara Bohorquez, Virginia Porras Hidalgo y Pilar Jurado Escamez.}

Servicio de Anatomía Patológica. Hospital Infanta Elena. Huelva. España.

Resumen.- OBJETIVO: El diagnóstico diferencial de masas escrotales incluye procesos inflamatorios, tumores malignos y lesiones traumáticas, incluyendo hematomas y hematocele crónico.

MÉTODOS/RESULTADOS: Presentamos dos casos de hematocele crónico discutiéndose las características clínicoradiológicas de dichas lesiones y su diagnóstico diferencial.

CONCLUSIONES: El diagnóstico preoperatorio de hematocele puede ser difícil, ya que la sintomatología puede imitar una lesión quística o una neoplasia.

Palabras clave: Hematocele. Masa testicular.
Concepción Lara Bohorquez

Servicio Anatomía Patológica

Hospital Infanta Elena

Ctra. Sevilla - Huelva s/n

21080 Hueva. (España)

clarab@andaluciajunta.es 
Summary.- OBJECTIVE: Differential diagnosis of a testicular mass includes inflammatory conditions, malignant tumors and traumatic lesions, including hematomas and hematocele.

METHODS/RESULTS: We report two cases of chronic hematocele. We discuss the clinico-radiologic characterisc and differential diagnosis.

CONCLUSION: Hematocele is difficult to diagnose preoperatively because its symptoms may mimic cysts or neoplasms.

Keywords: Hematocele. Testicular mass.

\section{INTRODUCCIÓN}

El testículo normal mide aproximadamente $5 \times 3 \times 3 \mathrm{~cm}$ de diámetro máximo, siendo la variabilidad bastante amplia. La estructura testicular adulta consta de dos túnicas testiculares: una cápsula que rodea al testículo o albugínea, que a su vez tiene tres estratos: el más externo es el mesotelio, el intermedio es una capa fibrocolágena densa y el interno es un conjunto de estructuras vasculares, a partir de la cual parten los septos que dividen al órgano en lóbulos; y una túnica más externa o vaginal también rodeada por mesotelio. Cada lóbulo tiene un complejo recorrido de túbulos seminíferos que empiezan y acaban en la rete testi, estos túbulos están rodeados por una pared tubular constituida por membrana basal y fibroblastos, y un epitelio formado por células de Sertoli y epitelio germinal, acompañadas de células de Leydig en el intersticio. Todas estas estructuras pueden desarrollar neoplasias, aunque la más frecuentes son las derivadas del epitelio germinal.

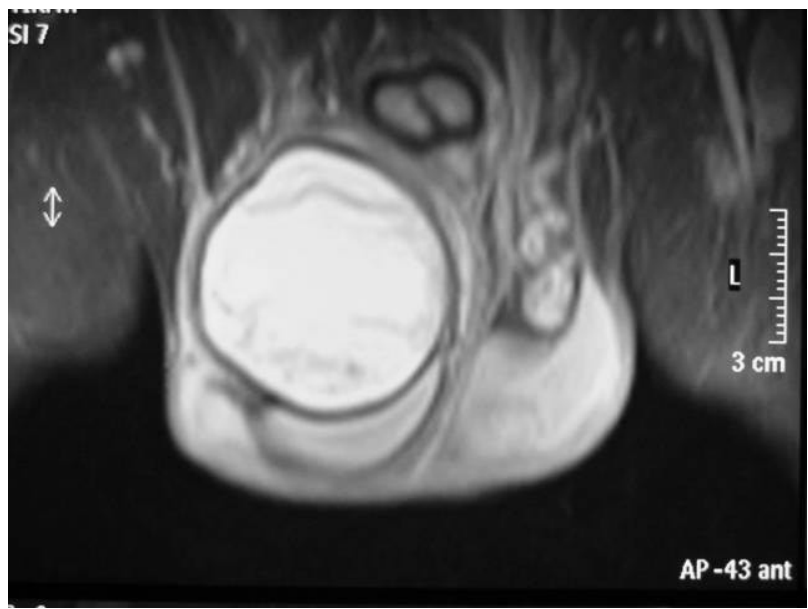

FIGURA 1. Lesión esférica de contornos bien definidos que comprime el parénquima testicular adyacente. RNM.
El espacio vaginal puede estar ocupado por líquido (hidrocele) o material hemático (hematocele), cuando este proceso se cronifica las cubiertas pueden fibrosarse y el líquido sufrir tabicación planteando problemas en el diagnóstico diferencial con verdaderas neoplasias desde el punto de vista clínico-radiológico.

Presentamos dos casos de hematocele crónico, planteando su diagnóstico diferencial y tratamiento.

\section{CASO CLÍNICO}

CASO 1: Paciente de 59 años de edad que acude a consulta de urología por aumento de tamaño escrotal derecho, sin antecedente de traumatismo. En el estudio de imagen (RNM), se describe una lesión focal esférica de contornos bien definidos por una fina pared fibrosa, hipointensa, y múltiples septos en su interior finos, aunque irregulares, tratándose de una lesión quística, de señal homogénea, de $6 \mathrm{~cm}$ de diámetro localizada en la cabeza del epidídimo derecho, que ejerce compresión extrínseca sobre el testículo ipsilateral, que queda deformado (Figura 1).

Con el diagnóstico de probable quiste septado de cabeza de epidídimo se procede a la intervención quirúrgica, realizándose exéresis completa de la lesión vía transescrotal.

Macroscópicamente se trata de una formación nodular, elástica, de superficie lisa y coloración pardusca. Al corte, se observa una cavidad multiquística, delimitada por una gruesa cápsula de aspecto fibroso, con múltiples tabiques de diverso grosor y ocupadas por material de aspecto hemático (Figura 2). En el estudio microscópico se confirma la naturaleza hemática del contenido y el componente fibroso de las paredes y septos, observán-

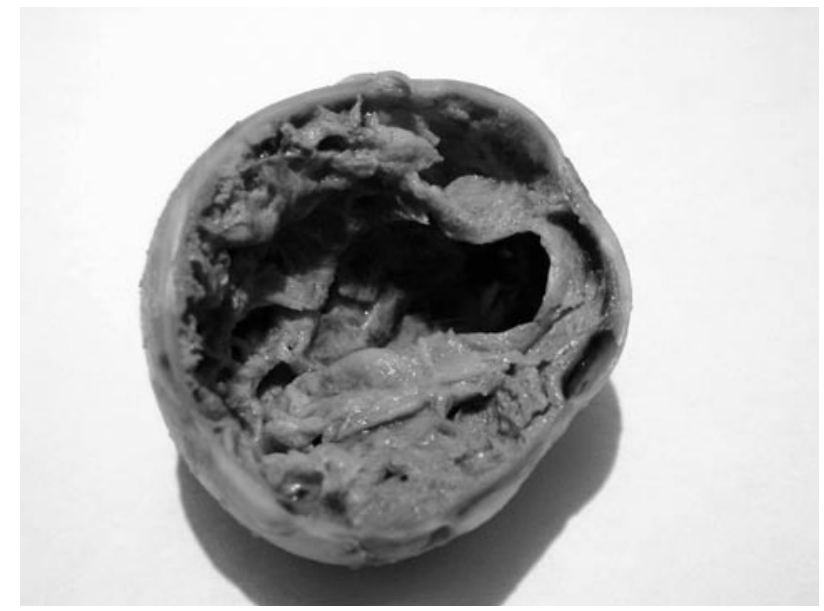

FIGURA 2. Formación nodular, de aspecto quístico, llena de material de pardusco, correpondiente a material hemático organizado. Fotografía macroscópica. 
dose en la cápsula imágenes de calcificación y granulomas de colesterol.

CASO 2: Paciente de 82 años de edad con antecedentes de hiperplasia benigna prostática, cardiopatía isquémica hipertensiva, EPOC avanzada, hiperuricemia e hipercolesterinemia, que acude a consulta por masa testicular derecha de un mes de evolución que ha aumentado de tamaño, sin fiebre y con marcadores tumorales negativos. En el estudio de imagen, ecografía, se describe una masa intratesticular con numerosos septos, que desplaza el parénquima testicular, de aproximadamente $5 \mathrm{~cm}$ de diámetro (Figura 3 ).

Con el diagnóstico de masa intratesticular derecha, se procede a la intervención quirúrgica realizándose orquiectomía radical.

En el estudio macroscópico de la pieza se describe una lesión de aspecto quístico, multilocular, ocupada por material de aspecto mucinoso, que alcanza un diámetro máximo de $6 \mathrm{~cm}$, y que desplaza y comprime el parénquima testicular adyacente, quedando situado a nivel de rete testi (Figura 4).

En el estudio microscópico la lesión está constituida por una gruesa cápsula fibrosa, con septos fibrosos acompañados de infiltrado inflamatorio crónico, con granulomas a colesterol, y abundante depósito de hemosiderina.

\section{DISCUSIÓN}

En el diagnóstico diferencial de masas escrotales se incluyen procesos inflamatorios, tumores malignos y lesiones traumáticas, incluyendo hematomas. El hematocele

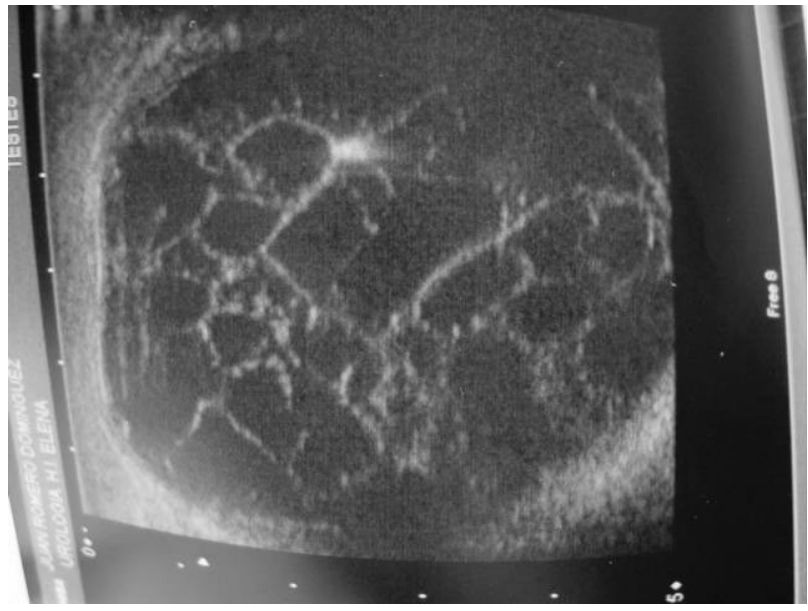

FIGURA 3. Masa quística con múltiples septos. Ecografía. resulta de la acumulación de sangre en el saco vaginal, presentando la mayoría de los pacientes un antecedente de traumatismo testicular.

Los hematoceles, en base a su etiología, pueden ser clasificados en secundarios e idiopáticos. Los idiopáticos son más frecuentes en personas de edad avanzada y la mayoría están en relación con traumatismos asintomáticos o infecciones; mientras que los secundarios no traumáticos suelen estar el relación con alteraciones hematológicas o vasculitis.

El diagnóstico preoperatorio de hematocele puede ser difícil, ya que la sintomatología a veces imita una lesión quística o una neoplasia. Desde el punto de vista de la exploración el testículo presenta una masa, pudiendo ayudar en el diagnóstico diferencial el estudio ecográfico, aunque no siempre es posible; no obstante, el hematocele debe tenerse en cuenta en el diagnóstico diferencial de una masa testicular multiquística.

Desde el punto de vista anatomopatológico los hallazgos corresponden a un hematoma organizado en el que pueden encontrarse calcificaciones, granulomas de colesterol en relación con la túnica vaginal y focos de metaplasia escamosa, existiendo algún caso aislado descrito en la literatura de carcinoma epidermoide sobre hematocele. Es aconsejable realizar un estudio minucioso de la lesión para excluir que exista una neoplasia relacionada que pueda pasar desapercibida por extensas áreas de necrosis y hemorragia.

El tratamiento de elección es cirugía conservadora con la evacuación completa del hematoma. En cuanto a las complicaciones, pueden presentar sobreinfección o necrosis del parénquima testicular adyacente por compresión o abscesificación.

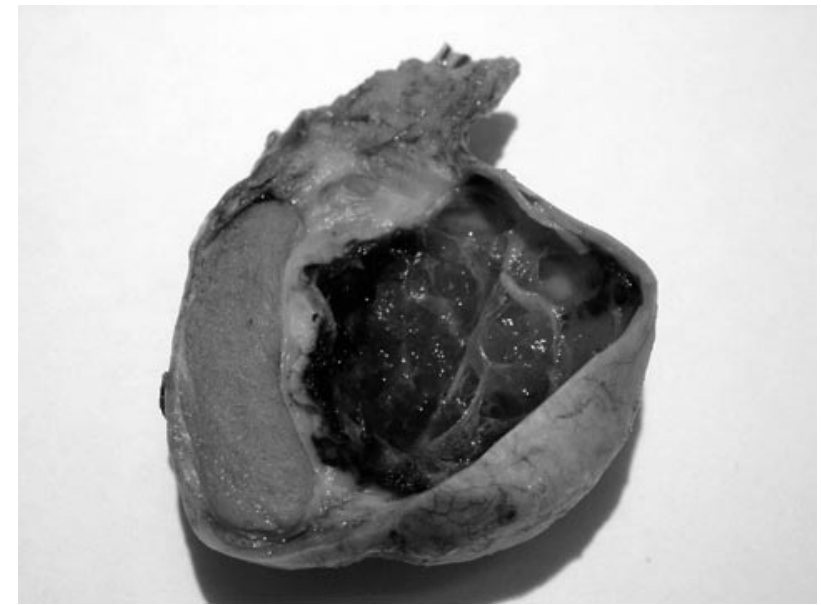

FIGURA 4. Pieza de orquiectomía con formación quística multilocular, que comprime el parénquima testicular adyacente. Fotografía macroscópica. 


\section{BIBLIOGRAFIA y LECTURAS RECOMENDADAS (*lectura de interés $y^{* *}$ lectura fundamental)}

**1. ALGABA, F.; MORENO, A; TRIAS, I.: "Uropatología tumoral". Capítulo 5. Tumores del testículo y sus anejos. pp 291. Grafos Press: Spain 1996.

*2. ALVAREZ-ALVAREZ, C.; FARINA-PEREZ, L.A.; BARROS, C.R.: "Idiopathic chronic hematocele of the vaginal sac". Int. Braz. J. Urol., 31: 555, 2005.

*3. SHAMSA, A.; KADKHODAYAN, A.; FEIZ-ZADEH, B. y cols.: "Testicular hematocele mimicking a testicular tumor: A case report and review of literature". Transplant Proc., 34: 2141, 2002.

4. MINAGAWA, T.; HIRABAYASHI, N.; FURUHATA, M. y cols.: "Two cases of an intrascrotal cystic mass mimicking a testicular tumor and review of the literature". Hinyokika Kiyo, 52: 311, 2006.

5. CUNNINGHAM, J.J.: "Sonographic fidings in clinically unsuspected acute and chronic scrotal hematoceles". ARJ Am. J. Roentgenol, 140: 749, 1983.

Casos Clínicos

Arch. Esp. Urol., 61, 4 (540-543), 2008

\section{ANGIOMIOLIPOMA EPITELIOIDE RENAL}

Igor Azurmendi Arin, Roberto Llarena Ibarguren, Jorge García-Olaverri Rodríguez, Ivan Olano Grasa, Emilio Cantón Aller y Carlos Pertusa Peña.

Servicio de Urología. Hospital de Cruces. Baracaldo. Vizcaya. España.
Resumen.- OBJETIVO: Poner de manifiesto la existencia de una variedad de angiomiolipoma, denominada epitelioide, con propiedades diferentes tanto histológicas como clínicas.

MÉTODO: Se presenta el caso de una mujer de 17 años, afecta de enfermedad de Bourneville, a la que en un control tomográfico renal se descubre la existencia de una masa sólida sugestiva de carcinoma, adyacente a otras de menor tamaño identificadas como angiomiolipomas.

RESULTADOS: Tras realizar varias tumorectomías, la sospechosa de carcinoma, de $4 \mathrm{~cm}$ de tamaño se diagnostica de angiomiolipoma epitelioide, con corroboración inmunohistoquímica mediante positividad para HMB45, y negatividad a vimentina y queratina.

CONCLUSIONES: A pesar de la posibilidad de coexistencia de adenocarcinoma y angiomiolipomas, no hay que descartar la existencia de la variedad epitelioide, sobre todo en pacientes con facomatosis. Los criterios quirúrgicos serán los mismos que para el resto de masas renales. Sin embargo, los criterios de seguimiento deberán ser más estrictos dada la posible evolución tórpida en cuanto a diseminación de esta infrecuente variedad de angiomilipoma.

Palabras clave: Angiomiolipoma. Epiteliode. Facomatosis.

Summary.- OBJECTIVE: To issue the existence of a variety of angiomyolipoma, named epithelioid, with different histological and clinic properties.

METHODS: We report the case of a 17-year-old female, with Bourneville's disease, who was discovered to have a solid renal mass suggestive of carcinoma in a control CT scan, adjacent to other smaller masses identified as angiomyolipomas.

RESULTS: After several tumorectomies, the suspicious mass, $4 \mathrm{~cm}$ in size, was diagnosed as epithelioid angiomyolipoma, with immunohistochemical confirmation of capacity for HMB45, and negative vimentin and keratin.

CONCLUSIONS: Despite the possibility of coexistence of adenocarcinoma and angiomyolipoma, the existence of an epithelioid variety cannot be discarded, mainly in patients with phakomatosis. The indications for surgery are the same than for the rest of renal masses. Nevertheless, follow-up criteria must be stricter due to the possibility of torpid outcome in terms of dissemination of this infrequent variety of angiomyolipoma.

Keywords: Angiomyolipoma. Epithelioid. Phakomatosis. 